\title{
Análise da produção científica sobre Gestão de Processos
}

\author{
Gustavo Trindade Choaire ${ }^{1}$ \\ João Victor Kothe ${ }^{l}$ \\ Júlia Weber Reuter ${ }^{2}$ \\ Karoline dos Santos ${ }^{3}$ \\ Juliana Ipê da Silva ${ }^{4}$ \\ Jaqueline de Moraes 5 \\ Marilei Bender Xavier ${ }^{6}$ \\ Liane Mählmann Kipper ${ }^{7}$
}

\section{RESUMO}

É notável o aumento de trabalhos científicos relacionados com a importância da tomada de decisão nas organizações, com a minimização de riscos e custos e com a maximização da chance de sucesso em empresas. Neste contexto, temas como a Gestão de Processos de Negócio, a Análise de Decisão Multicritério e a Teoria da Utilidade Multiatributo são fundamentais para o entendimento desta importância. Assim, foi elaborado um estudo bibliométrico a respeito destes temas, que foram pesquisados no período de 1970 a 2015 e mostram a evolução dos mesmos nas pesquisas científicas. O tópico Análise de Decisão Multicritério apresentou 24,6\% dos 4334 artigos, o tópico Teoria da Utilidade Multiatributo, 1,2\% e a Gestão de Processos de Negócio apresentou 74,2\% do número de publicações, o que demonstrou ser o tópico mais tratado pelos escritores e, consequentemente, o que desperta maior interesse entre os mesmos no período analisado.

Palavras-chave: Bibliometria. Gestão de Processos de Negócio. Análise de Decisão Multicritério. Teoria da Utilidade.

\begin{abstract}
It is notable the increase of scientific work related to the importance of decision making in organizations, minimizing risks and costs and maximizing the chance of success in companies. In this context, topics such as Business Process Management, Multicriteria Decision Analysis and Multi-Attribute Utility Theory are fundamental to understanding this importance. Consequently, a bibliometric study was elaborated on these topics. These topics were researched in the period from 1970 to 2015 and show the evolution of them in scientific research. The Multiple Criteria Decision Analysis presented 24.6\% of the 4334 articles, the topic Multiple Attribute Ultility Theory, 1.2\% and Business Process Management presented

\footnotetext{
${ }^{1}$ Alunos do Curso de Engenharia Mecânica da Universidade de Santa Cruz do Sul - UNISC.

${ }^{2}$ Aluna do Curso de Engenharia Civil da Universidade de Santa Cruz do Sul.

${ }^{3}$ Aluna do Curso de Engenharia de Produção da Universidade de Santa Cruz do Sul.

${ }^{4}$ Aluna do Curso de Engenharia Química da Universidade de Santa Cruz do Sul.

${ }^{5}$ Mestranda do Programa de Pós-Graduação em Sistemas e Processos Industriais da Universidade de Santa Cruz do Sul.

${ }^{6}$ Professora Doutora em Sensoriamento Remoto.

${ }^{7}$ Professora Doutora do Programa de Pós-Graduação em Sistemas e Processos Industriais da Universidade de Santa Cruz do Sul - UNISC.<liane@unisc.br>
} 
$74.2 \%$ of the number of publications, which proved to be the most treated topic by writers and consequently the one which arouses greater interest among them.

Keywords: Bibliometry. Bussiness Process Management. Multiple Criteria Decision Analysis. Multiple Attribute Utility Theory.

\section{INTRODUÇÃO}

No cenário atual observa-se, através de pesquisas em bases de dados, um aumento de trabalhos científicos relacionados com a importância da tomada de decisão, com a minimização de riscos e custos e com a maximização da chance de sucesso em empresas. Diante do mercado concorrido e competitivo, é imprescindível o uso de ferramentas e métodos que auxiliem gestores nas tomadas de decisões, para que as mesmas sejam precisas e estratégicas. Segundo Guitouni e Martel (1998), o método de múltiplo critério deixou de ser aquele focado em aperfeiçoar um único objetivo em meio a várias possíveis soluções, passando àquele em que muitos conflitos são levados em consideração e a solução tomada não é mais aquela opcional, mas sim, a que satisfaz todos os critérios que geram conflito.

Juntamente com a análise de múltiplos critérios, a Gestão de Processos de Negócio (BPM - Bussiness Process Management) estabelece uma importância similar para as empresas que buscam atingir melhores resultados. Os principais conceitos desenvolvidos na revolução industrial são fundamentais nos sistemas de gestão atuais segundo Van Der Aalst (2013). Ainda segundo o autor, com o surgimento das inovações no ramo da computação e da comunicação, tornou-se cada vez mais complexo de gerir esses processos através de instruções e configurações precisas, que consequentemente irão gerar resultados positivos.

Outro tipo de método de análise de múltiplos critérios é a Teoria da Utilidade. Kapur, Singh e Singh (2015) consideram interessante o uso deste método na escolha de um carro ou de um trabalho. Gomes (1998) afirma que esta teoria demonstra a representação das preferências relativas de um indivíduo entre os elementos de um conjunto e utiliza números reais para representá-los.

Em ambos os casos, a melhor decisão das alternativas resulta em múltiplos atributos ou critérios. Para resolver o problema apresentado por Kapur, Singh e Singh (2015), é sugerido o uso da teoria da utilidade que auxilia na melhor decisão excluindo os fatores conflitantes de forma simultânea. 
Diante do exposto, o trabalho tem por objetivo a elaboração de uma pesquisa bibliométrica com enfoque na análise dos três temas relevantes e que inferem na indústria mundialmente, sendo eles, a Gestão de Processos de Negócio, a Análise de Decisão Multicritério e a Teoria da Utilidade. Estes temas foram pesquisados no período de 1970 a 2015 e mostram a evolução dos mesmos nas pesquisas científicas.

\section{FUNDAMENTAÇÃO TEÓRICA}

A bibliometria busca analisar e elaborar indicadores através de métodos estatísticos e matemáticos sobre determinadas áreas, revistas científicas, jornais, organizações ou países. Ferreira (2010) afirma que em 1934 foi criado o termo Bibliometria por Paul Otlet, no Tratado da Documentação, antes sendo uma ciência conhecida por "bibliografia estatística", este criado por Hulme em 1923. Porém, consolidou-se o termo bibliometria após a publicação do artigo com o título "Bibliografia estatística ou Bibliometria?" em 1969 por Pritchard.

Sendo assim, o termo surgiu da necessidade de estudar e avaliar as atividades de produção e comunicação científica. A bibliometria procura um perfil dos registros do conhecimento, servindo-se de um método quantificável.

De acordo com Araújo (2006), analisando-se os dados apenas através de citações, já é possível classificar os autores mais citados, os mais produtivos, o fator de impacto dos mesmos, a procedência geográfica e/ou institucional dos autores e da bibliografia utilizada e os periódicos mais citados.

A Gestão de Processos de Negócios, conhecida como BPM, auxilia não só o desempenho organizacional, mas também visa obter como resultado uma melhora nas atividades colaborativas internas (PRADABWONG et al., 2015). Segundo Tadeu de Oliveira Lacerda (2015), a Gestão de Processos de Negócios é um processo que visa criar e manter uma vantagem competitiva em organizações contemporâneas.

De acordo com Ogle, Dee \& Cox (2015), o Multi-Attribute Utility Theory (MAUT) é uma técnica analítica baseada na análise de decisão que permite considerar sistematicamente julgamentos de valor de múltiplos objetivos concorrentes. A principal característica é o uso de funções de utilidade para modelar atributos de medidas.

Para Convertino et al. (2013), a técnica MCDA (Multi Criteria Decision Analysis) deve ser usada quando há muitos interessados em algum projeto/trabalho, tendo muitos objetivos e alternativas para considerar. Também segundo o autor, a Teoria da Utilidade (MAUT) está diretamente relacionada com a Análise Multicritério (MCDA), pois segundo ele, o MAUT é um método pertencente à análise multicritério. 
Minatour, Bonakdari e Aliakbarkhani (2016), exemplificam que quando os métodos de análise multicritério não conseguem lidar com informações imprecisas, um instrumento de análise derivado do método é introduzido, os conjuntos difusos. A lógica difusa aumenta a confiabilidade no gerenciamento dos processos de negócio, além de evitar que as organizações paguem custos adicionais (BAGHERZADEH et al., 2016).

As relações entre os métodos derivados da análise multicritério e suas aplicações na gestão de processos tornaram interessante a realização de uma bibliometria para comprovar a convergência dos termos.

\section{MATERIAIS E MÉTODOS}

O estudo foi exploratório junto à base de dados Scopus (Elsevier), a maior base de dados de resumos e citações da literatura peer-reviewed: revistas científicas, livros e anais de conferências; para a realização da coleta de dados. As etapas metodológicas deste trabalho estão representadas na Figura 1.

\section{Figura 1 - Etapas metodológicas da pesquisa}

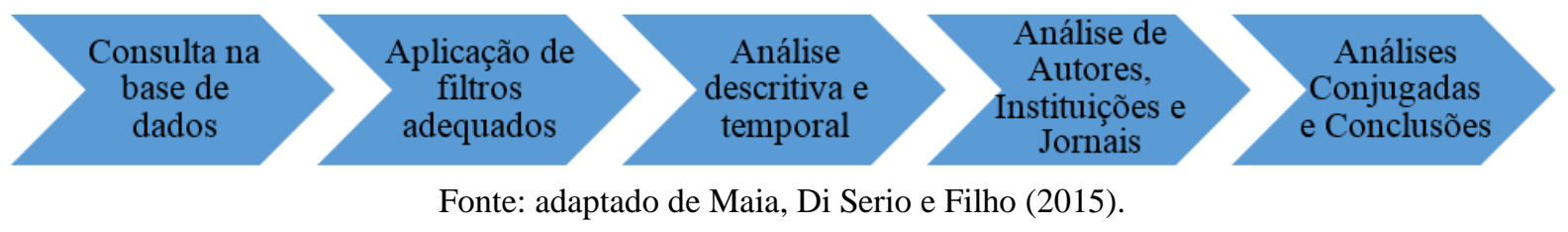

A consulta na base de dados ocorreu pela página da biblioteca da UNISC (http://www.unisc.br/pt/servicosonline/biblioteca/biblioteca-virtual/base-de-dados), tendo sido pesquisados os seguintes termos de busca: "business process management", "multiple criteria decision analysis" e "multiple attribute utility theory";no período de 1970-2015 e foram considerados para a análise dos dados apenas artigos. A aplicação dos filtros seguiu os seguintes critérios: ano de publicação, autor, instituições, países com mais publicações e jornais. Foram pesquisados métodos de análise e tomada de decisões que influenciam quatro diferentes áreas do conhecimento, sendo elas, Life Sciences, Health Sciences, PhysicalSciences e Social Sciences \& Humanities.

Para a realização da análise dos dados, foram construídas planilhas no software Excel para a análise descritiva dos resultados. Para avaliar o desempenho dos autores referentes a cada tópico foi utilizado o software VOSviewer, que pode, por exemplo, ser empregado na construção de mapas de autores ou revistas com base em dados de cocitação ou para construir mapas de palavras-chave com base em dados de co-ocorrência. Segundo Lucas e Garcia- 
Zorita (2014) o método de cocitação unifica os artigos citados pelo mesmo documento. O programa oferece um visualizador que permite que mapas bibliométricos sejam examinados em detalhes (VAN ECK; WALTMAN, 2010). A seguir estão apresentados e discutidos os resultados encontrados.

\section{RESULTADOS}

A primeira consulta à base de dados Scopus forneceu um total de 58.368 documentos. Considerando apenas os artigos, o número de documentos fícou em 4.334, ou seja, 7,425\% dos documentos encontrados. Como resultados, observou-se que o termo de busca Multiple Criteria Decision Analysis apresentou 24,6\% dos 4.334 artigos, o termo Multiple Attribute Ultility Theory, 1,2\% e Business Process Management apresentou 74,2\%, que demonstrou ser o tópico mais tratado pelos escritores e, consequentemente, o que desperta maior interesse entre os mesmos. Na sequência são detalhados os resultados específicos da pesquisa.

\subsection{Ano de Publicação}

Apresenta-se, na sequência, a Figura 2 que destaca a evolução das publicações dos temas pesquisados durante os anos, com destaque para a Gestão de Processos, tema que possui o maior número de artigos publicados.

Figura 2 - Número de publicações dos temas pesquisados no período de 1970 a 2015

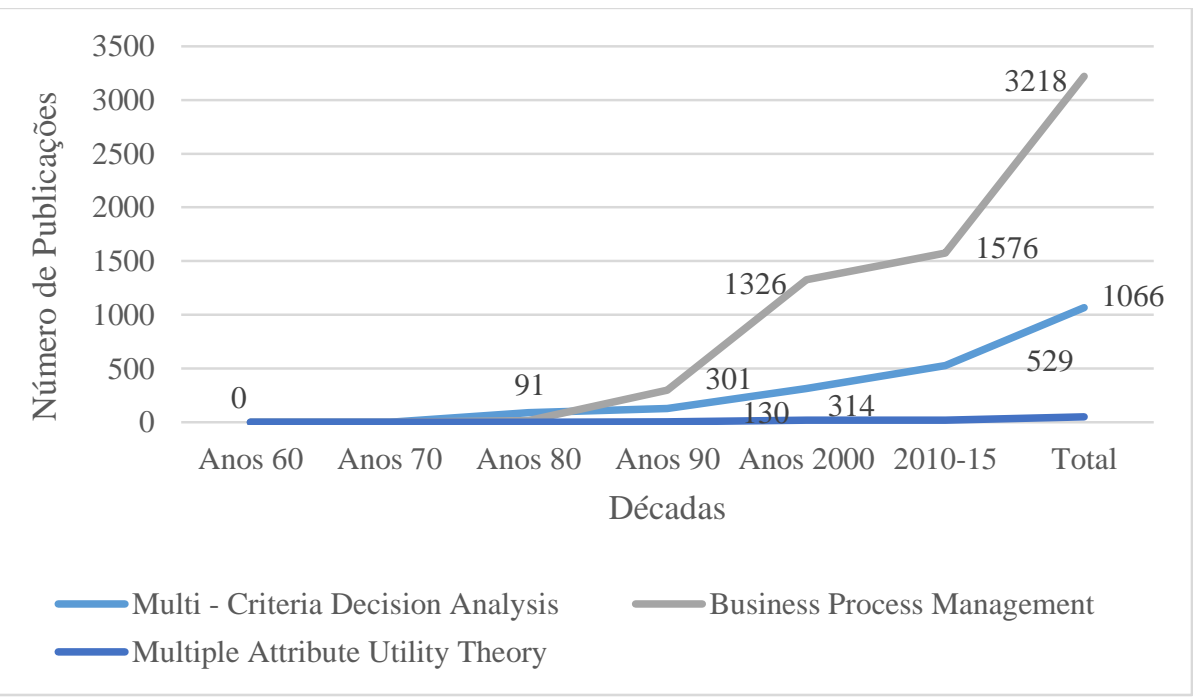

Fonte: elaborado pelos autores (2016). 
Durante os anos 60 não foram encontradas publicações científicas relacionadas aos tópicos pesquisados, mas já na década seguinte a presença de dois artigos na área de análise multicritério comprovou o surgimento do estudo sobre tal tópico. As publicações a respeito dos três tópicos cresceram durante os últimos anos e esse crescimento foi possível de ser observado a partir dos anos 90.

A Teoria da Utilidade foi o tópico que apresentou o menor crescimento, quase inexpressivo se comparado com a Análise Multicritério e com a Gestão de Processos, apresentando apenas duas publicações durante as décadas de 70 e 80, sendo assim excluída da análise bibliométrica. Nota-se, também, que no decorrer dos anos 90 a produção sobre os tópicos de Análise Multicritério e Gestão de Processos eram bem próximas, mas a partir da virada do século houve uma dispersão muito grande da produção de ambas. Na área de Análise Multicritério a produção de artigos duplicou, enquanto que em relação à Gestão de Processos as publicações quadruplicaram.

Na Figura 3 é possível observar que em algumas décadas a Análise Multicritério era o tópico mais citado entre os que foram pesquisados, com o seu pico de citações durante o início do século XXI.

\section{Figura 3 - Número de citações de cada tema durante o período pesquisado}

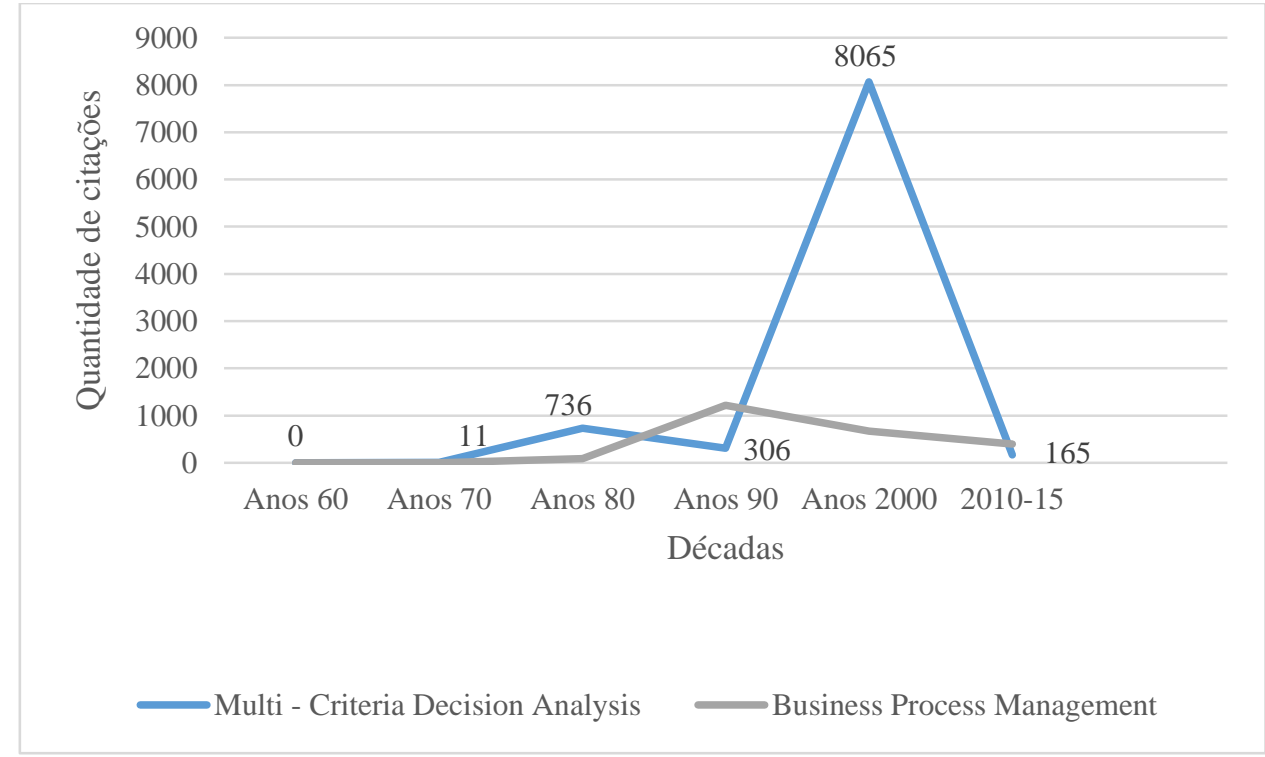

Fonte: elaborado pelos autores (2016).

A Gestão de Processos teve seu pico durante os anos 90 e, após tal período, começou a diminuir, diferentemente da Teoria da Utilidade, que obteve um pequeno crescimento nas citações e começou a diminuir na virada do século. 


\subsection{Desempenho dos autores}

As imagens das Figuras 4 e 5 foram criadas a partir de cocitações e autores mais citados. O número mínimo de citações de um mesmo autor foi determinado como 20 e o resultado para cada tópico pesquisado apresentou uma variação, pois depende do número de publicações exibidos na base de dados.

Para a gestão de processos, Figura 4, o número de autores que se encaixaram no padrão pré-determinado foi de 500. Já na análise multicritério, Figura 5, esse número diminuiu para 299 e na teoria da utilidade quase inexpressivos, caindo para 9 autores.

\section{Figura 4 - Resultado de cocitações entre autores sobre o tema Gestão de Processos de Negócio}

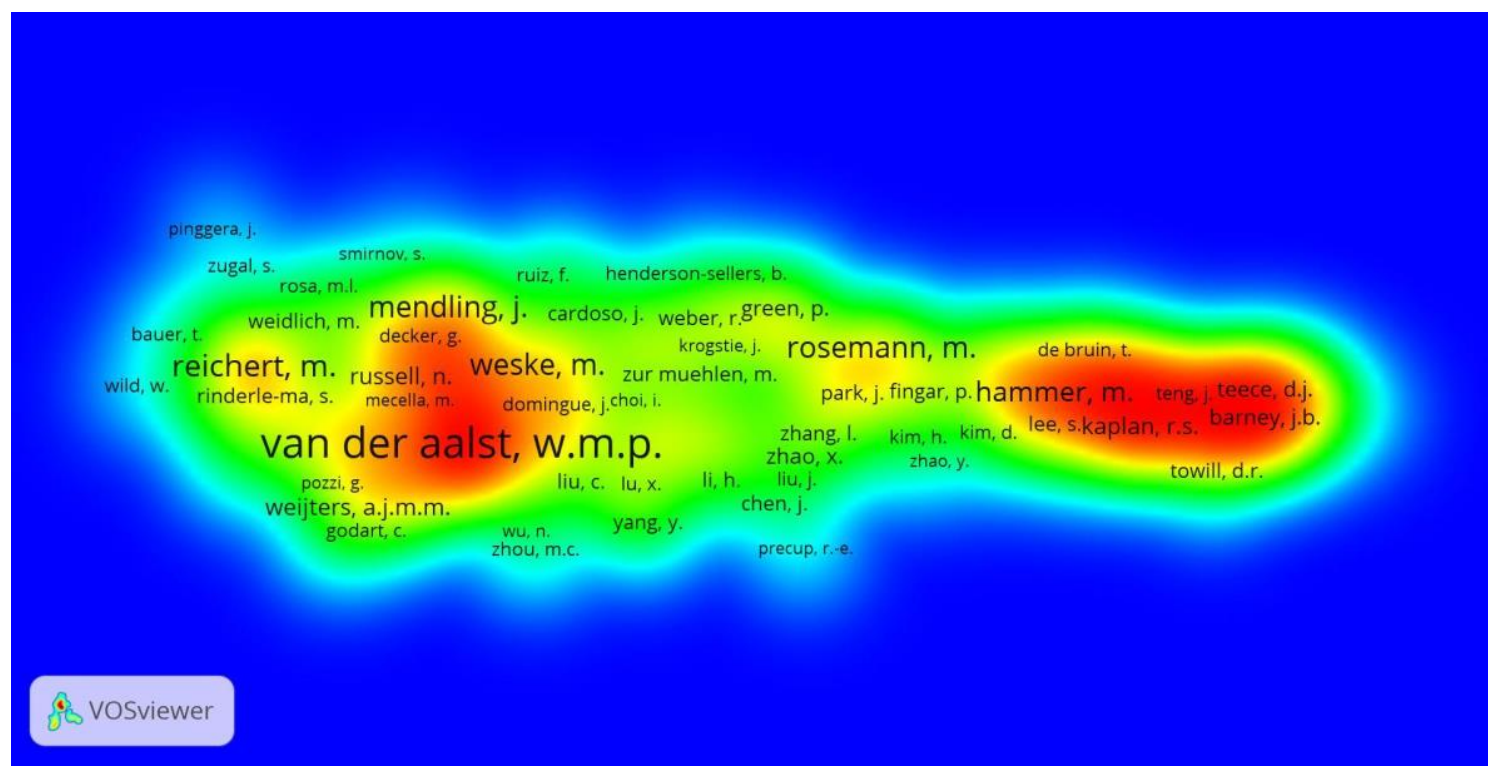

Fonte: elaborado pelos autores (2016).

É possível observar que quanto maior o tamanho da fonte do nome de cada autor, maior é o número de vezes que ele foi citado. Dessa forma, Wil van der Aalst é o autor que possui o maior número de artigos publicados e também é o autor mais citado quando se trata da Gestão de Processos. Porém, é importante destacar que nem sempre os autores com o maior número de publicações são os mais citados. É o caso de Hye-Rim Bae, autor que fica logo após Wil van der Aalst no número de publicações, mas não é um destaque quando se trata de citações.

Essa imagem também possibilita a visualização de áreas com cores mais fortes e mais fracas. Por exemplo, o vermelho é uma cor mais forte e que demonstra uma maior 
concentração de autores acerca de um tópico específico na Gestão de Processos. Já o verde, uma cor mais fraca, demonstra uma concentração pequena de autores acerca de um tópico específico menos estudado ou menos citado.

Também é possível verificar a presença de clusters. Porter (1998) caracteriza cluster como concentrações geográficas de empresas e instituições interconectadas. Clusters incluem universidades e associações comerciais que oferecem treinamento especializado como educação, informação, pesquisa e suporte técnico. É possível observar na Figura 4 a presença muito próxima dos variados clusters, ou seja, uma grande inter-relação entre as publicações de diferentes autores. No cluster que o autor Wil van der Aalst é referência por ser o mais citado, o enfoque das pesquisas publicadas na base de dados pelos demais autores, consiste em apresentar e explanar o conceito do método da Gestão de Processos de Negócio.

\section{Figura 5 -Mapa de densidade do resultado de cocitações entre autores sobre o tópico Análise Multicritério de Decisão}

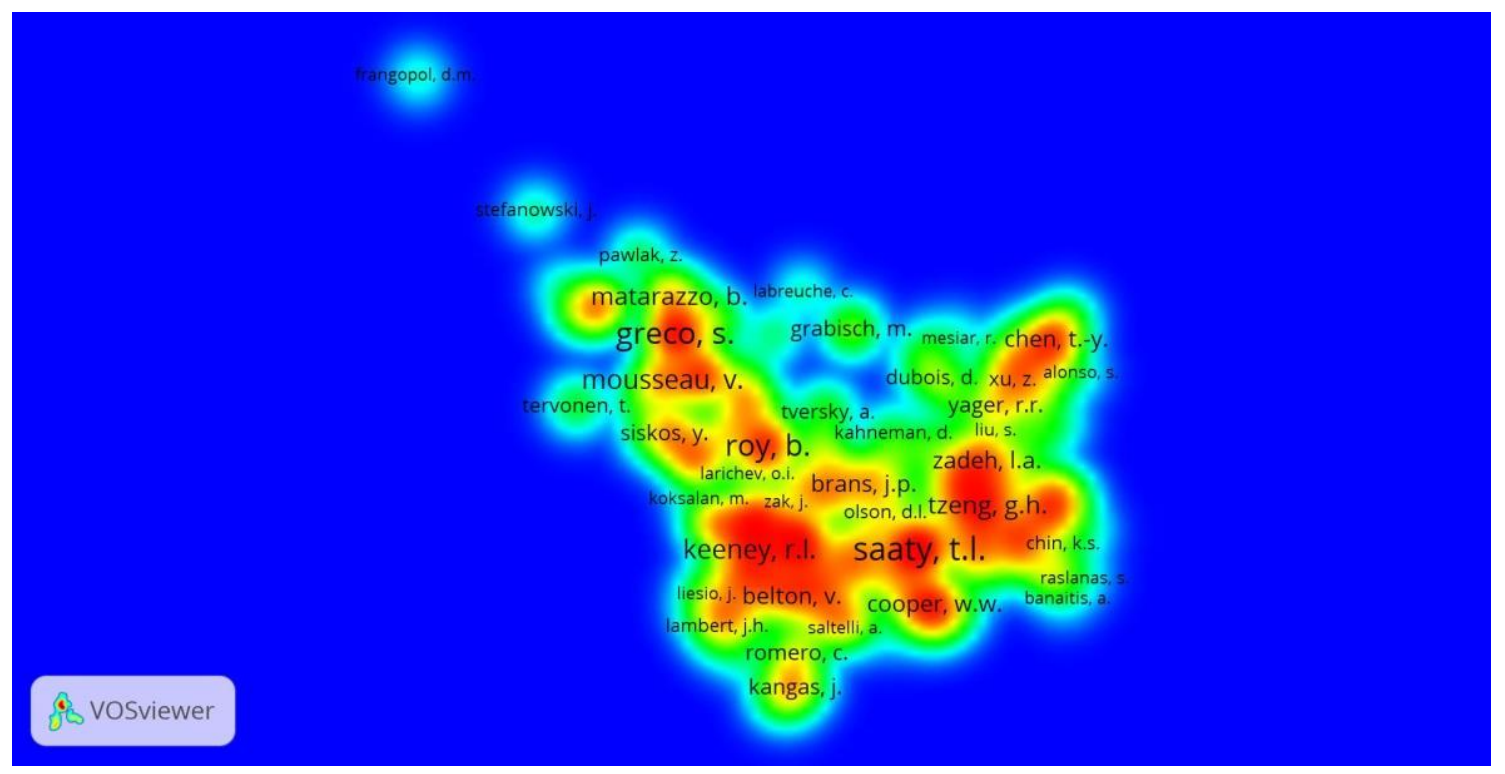

Fonte: elaborado pelos autores (2016).

$\mathrm{Na}$ Figura 5 é possível observar que, assim como na Figura 4, as cores e o tamanho da fonte caracterizam a cocitação entre autores sobre o tema Análise Multicritério de Decisão. Sendo assim, Saaty, T.L. destaca-se como autor com mais citações, porém não se destaca em número de publicações. O autor que ocupa esta posição é Chen, T.Y., mas em números de citações não se sobressai. Logo atrás de Chen, T.Y., está o autor Greco, S. nos números de publicações, tendo pouco destaque em números de citações.

Já a Figura 6 possibilita a visualização dos clusters, cada um representado por uma 
cor diferente.

Figura 6 - Resultado de relações de cocitações entre autores sobre o tema Análise Multicritério de Decisão

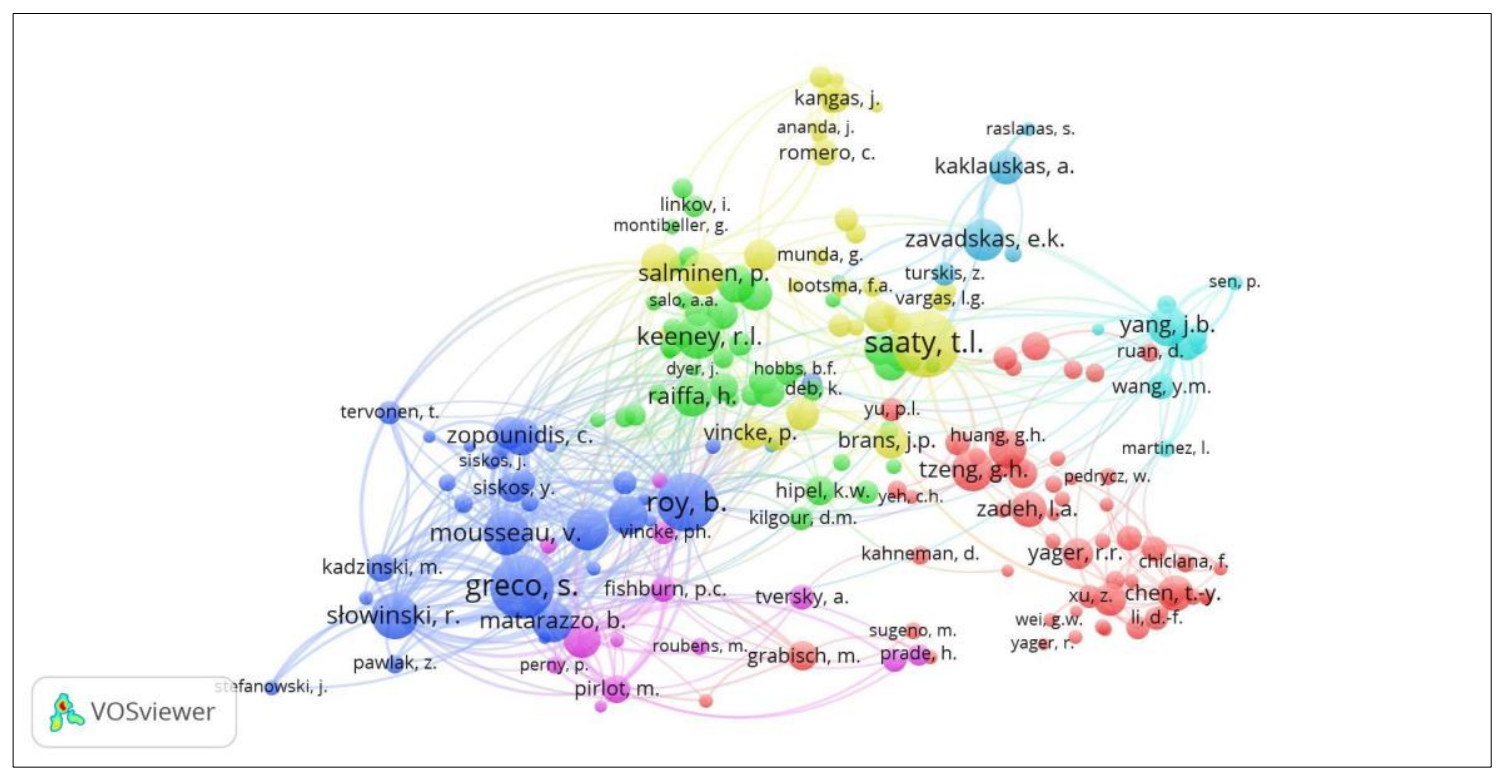

Fonte: elaborado pelos autores (2016).

Cada cluster possui um ou mais autores como referência no assunto que está sendo tratado naquele agrupamento específico. A Tabela 1 apresenta a relação sobre o assunto tratado pelos autores e a qual cluster (representado pelas cores) os mesmos pertencem. Os dados foram obtidos através da pesquisa sobre os trabalhos publicados pelos autores.

Tabela 1 - Autores e assuntos tratados em diferentes clusters

\begin{tabular}{|c|c|c|}
\hline Autores & Cor do cluster & Assunto \\
\hline GRECO, S. & Azul escuro & Value -focusedthinking \\
\hline $\begin{array}{c}\text { KEENEY, } \\
\text { R.L. }\end{array}$ & Verde & AHP, ANP \\
\hline $\begin{array}{c}\text { SAATY, } \\
\text { T.L. }\end{array}$ & Amarelo \\
\hline $\begin{array}{c}\text { TZENG, } \\
\text { G.H. }\end{array}$ & Vermelho & VIKOR, TOPSIS, DEMATEL \\
\hline PIRLOT, M. & Rosa & TABU, ELECTRE, algoritmos \\
\hline YANG, J.B. & Azul claro & FUZZY focado em engenharia \\
\hline \multicolumn{2}{|c|}{ Fonte: elaborado pelos autores (2016). }
\end{tabular}




\subsection{Desempenho das instituições}

O tópico sobre o desempenho das instituições tem sido um dos assuntos mais discutidos nos últimos anos. Segundo Klannet al. (2012), de indicadores totalmente financeiros, e que incluem um conjunto de indicadores que não contêm informações financeiras, a "discussão" gira em torno de quais indicadores seriam mais adequados para verificar o desempenho das organizações.

Na Tabela 2 foram pesquisados os números de publicações e o país de origem de cada instituição, de acordo com o tópico em questão.

Tabela 2 - Instituições com maior número de publicações

\begin{tabular}{|c|c|c|c|}
\hline Ranking & $\begin{array}{l}\text { Número de } \\
\text { Publicações }\end{array}$ & Instituição & $\begin{array}{l}\text { País de } \\
\text { Origem }\end{array}$ \\
\hline 1 & 74 & Technische Universiteit Eindhoven & Holanda \\
\hline 2 & 53 & Tsinghua University & China \\
\hline 3 & 48 & Queensland University of Technology & Austrália \\
\hline 4 & 44 & Universityof Ljubljana & Eslovênia \\
\hline 5 & 30 & Aalto University & Finlândia \\
\hline 6 & 30 & Chang Gung University & Taiwan \\
\hline 7 & 27 & Universita degli Studi di Catania & Itália \\
\hline 8 & 26 & Hong Kong Polytechnic University & Hong Kong \\
\hline 9 & 24 & Politechnika Poznanska & Polônia \\
\hline 10 & 24 & Vilniaus Gedimino Technikos Universitetas & Lituânia \\
\hline
\end{tabular}

Fonte: elaborado pelos autores (2016).

É possível observar na Tabela 2 que, quando se trata de gestão de processos, a área com o maior número de artigos publicados é de duas instituições asiáticas entre as cinco primeiras que possuem mais publicação. Tsinghua University (China) e University of Ljubljana (Hong Kong), representam 39,6\% das cinco primeiras instituições que mais publicaram, com um total de 97 documentos. A universidade que lidera esse ranking das cinco primeiras está situada na Holanda e representa 74 publicações, $51 \%$ de todas as publicações da Holanda.

$\mathrm{Na}$ área de análise multicritério duas instituições lideram com o mesmo número de publicações. A Chang Kung University (Taiwan) e a Aalto University (Finlândia), cada uma com possui 30 documentos publicados. Diferentemente da gestão de processos, o que predomina agora nas cinco primeiras instituições com mais publicações são instituições 
europeias. Somente a Aalto University representa $60 \%$ das publicações finlandesas neste tópico.

\subsection{Desempenho dos países/territórios}

Sabe-se que os Estados Unidos é uma grande potência mundial, que dá uma atenção especial à educação, o que é facilmente perceptível ao realizar pesquisa e também por ocupar uma das posições mais altas no ranking. Logo atrás dos Estados Unidos, está a China, caracterizada pela sua crescente economia, mas também pelo seu crescimento na área da educação, com ênfase na questão científica. Levando-se em conta o número de publicações de cada país, é possível observar na Figura 7 que, em número de publicações, os Estados Unidos estão à frente das publicações dos demais países sobre o tema Gestão de Processos.

\section{Figura 7 - Número de publicações de cada país sobre o tema Gestão de Processos}

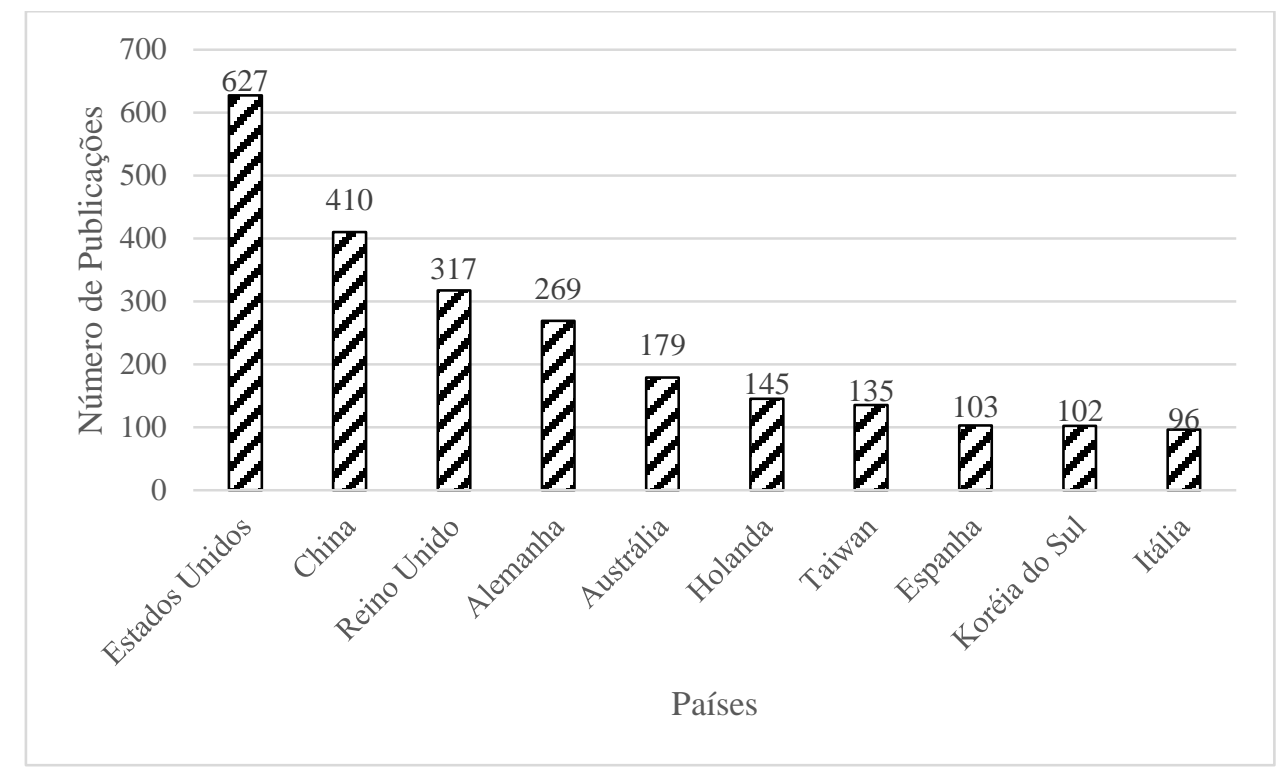

Fonte: elaborado pelos autores (2016).

É possível observar na Figura 8 que, em relação ao número de publicações sobre o tema Análise Multicritério, os EUA mantêm-se como o país líder, havendo uma alteração entre as colocações seguintes. A China deixa de ocupar a segunda colocação, dando lugar para Taiwan. 
Figura 8 - Número de publicações de cada País sobre o tema Análise

\section{Multicritério}

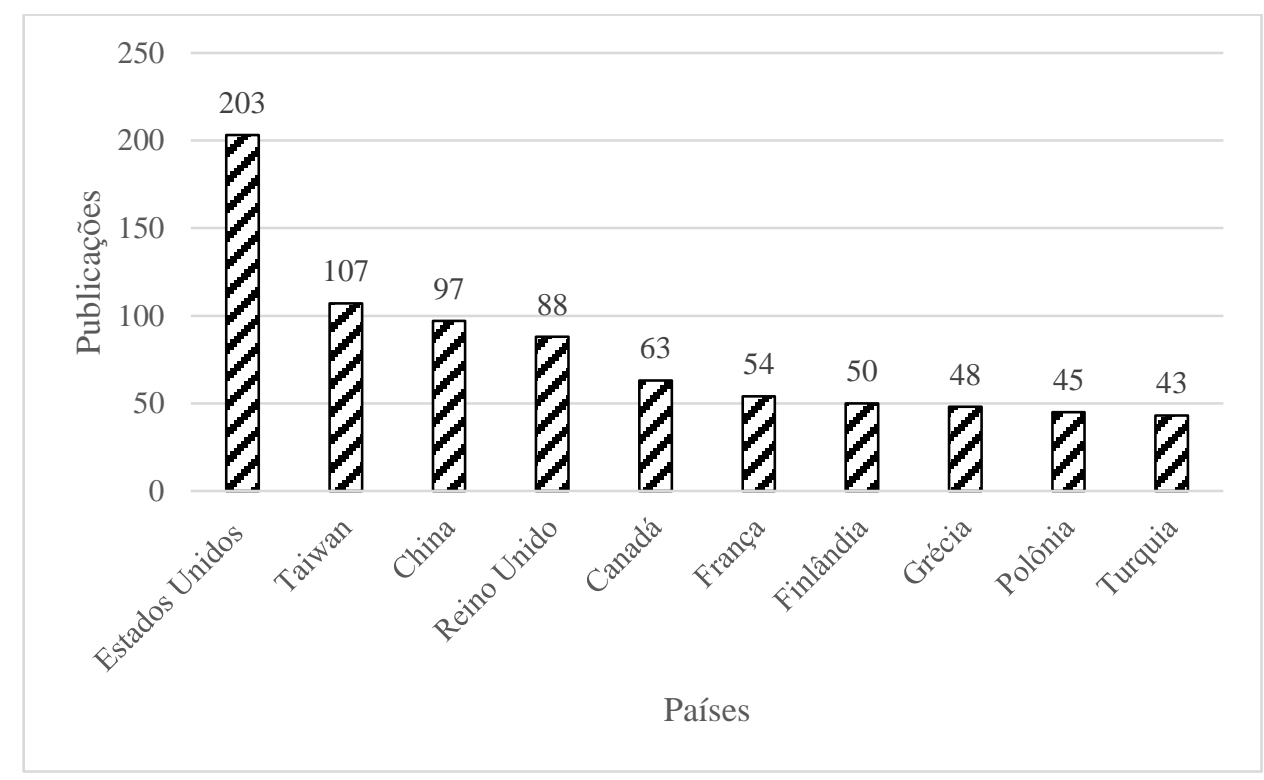

Fonte: elaborado pelos autores (2016).

A Teoria da Utilidade foi o tópico que apresentou o menor crescimento, quase inexpressivo se comparado com a Análise Multicritério e com a Gestão de Processos, com apenas duas publicações durante as décadas de 70 e 80 . Devido ao número ínfimo de publicações sobre a teoria da utilidade, a mesma foi excluída da análise mais aprofundada por não apresentar artigos publicados a partir dos anos de 1980.

\subsection{Desempenho dos Jornais}

Este item aborda a temática dos jornais, com foco na geração de clusters para tornar possível a visualização dos temas que se destacam em popularidade e estão difundidos nas buscas e pesquisas em periódicos.

A Figura 9 apresenta um mapa bibliométrico elaborado a partir de um acoplamento bibliográfico entre os jornais que publicam sobre a Gestão de Processos. Segundo Da Silveira Guedes (2012), o acoplamento bibliográfico é um procedimento que visa analisar retrospectivas citações que aparecem comumente em artigos diferentes, análise feita geralmente através das referências. Cinco clusters foram identificados, mas apenas dois demonstraram maior importância.

No cluster representado pela cor verde, o periódico Business Process Management Journal é o que lidera o segmento e possui artigos indexados que tratam sobre metodologias, 
técnicas e ferramentas sobre a modelagem da Gestão de Processos, assim como inúmeros estudos de casos focados em Gestão de Processos de Negócio.

Por outro lado, o agrupamento referente à cor vermelha é composto por jornais que indexam artigos e trabalhos referentes a sistemas de informação para empresas e aplicação industrial para desenvolvimento de softwares, além de pesquisas sobre aspectos econômicos e comerciais dos processos de desenvolvimento de software. São exemplos desse tipo de pesquisa os jornais Journal of Systems and Software, Lecture Notes in Business Information Processing e Jisuanji Jicheng Zhizao Xitong / Computer Integrated Manufacturing Systems.

\section{Figura 9 - Desempenho dos jornais sobre Gestão de Processo}

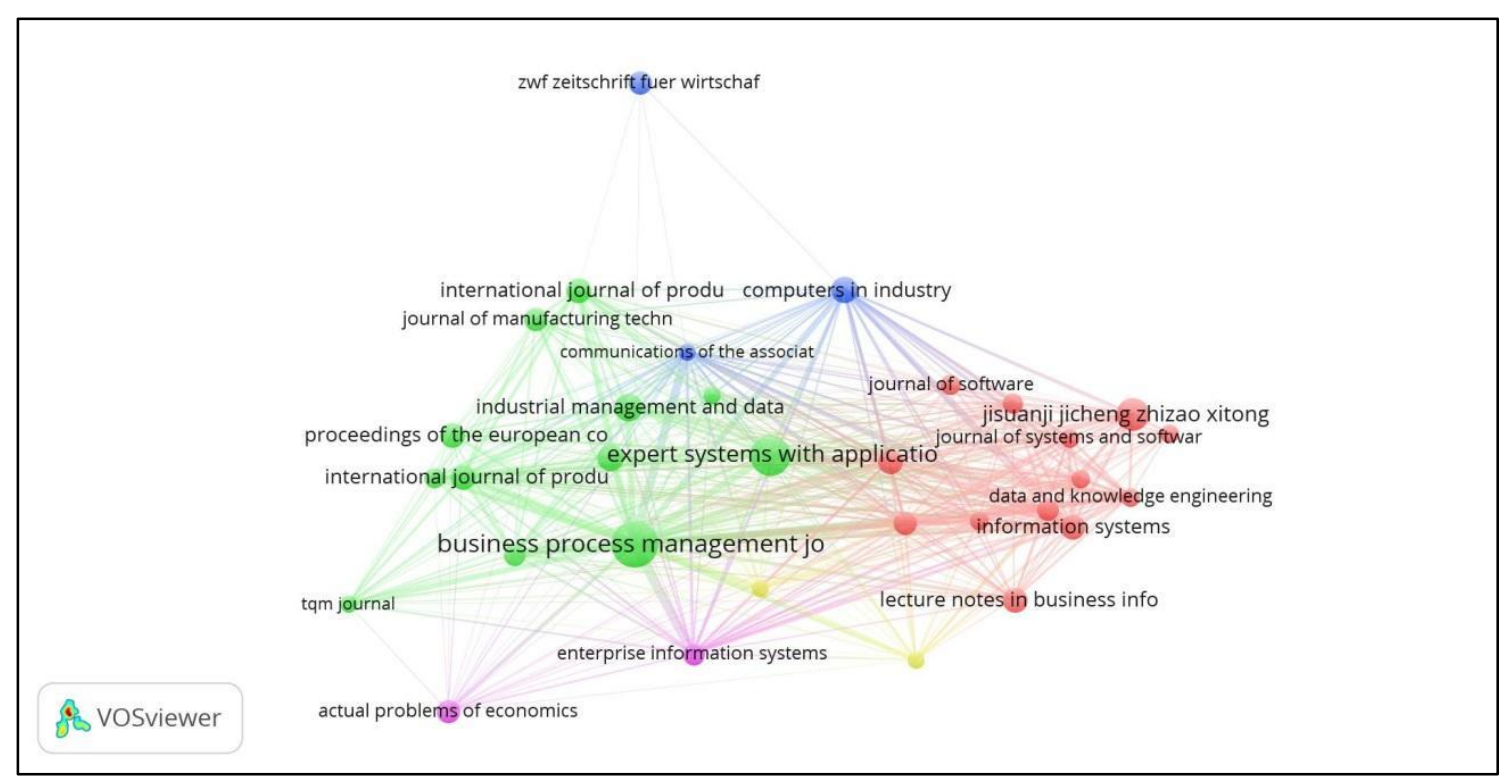

Fonte: elaborado pelos autores (2016).

Na Figura 10, o acoplamento bibliográfico foi realizado com enfoque no tema da Análise Multicritério, onde foram identificados também cinco clusters, mas diferente da Gestão de Processos, apenas um grupo apresentou maior importância e relevância.

Esse grupo é representado pela cor vermelha e possui jornais como o European Journal of Operational Research, Journal of the Operational Research Society e International Journey of Production Research. Os periódicos que estão interligados nesse agrupamento indexam artigos que tratam dos mesmos assuntos, produzindo trabalhos com enfoque em contribuir para a metodologia da pesquisa operacional e das práticas de tomada de decisão, assim como técnicas fundamentais desenvolvidas em ciências computacionais, de decisão e matemáticas, que podem ser utilizadas na concepção, medição ou operação de 
sistemas de produção e logística.

Figura 10 - Desempenho dos jornais sobre Análise Multicritério

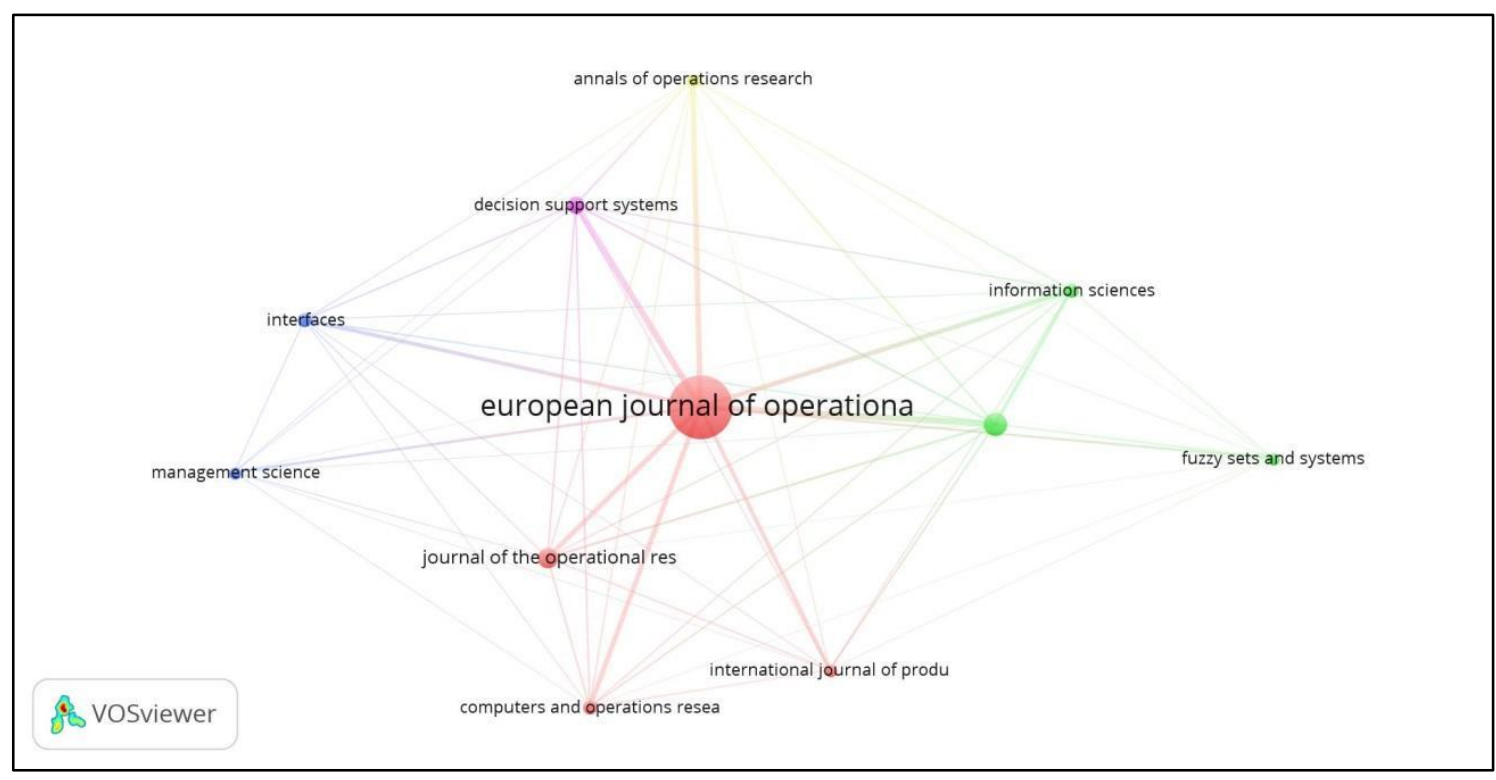

Fonte: elaborado pelos autores (2016).

\section{CONCLUSÃO}

Através da análise dos gráficos e imagens geradas pelos softwares VOSviewer e Excel, bem como da leitura descritiva das observações, verificou-se que a Gestão de Processos está sendo a mais pesquisada dentre os três temas estudados. As hipóteses para este avanço levam em consideração que as empresas estão impulsionando as pesquisas nessas áreas, devido aos benefícios que os resultados das pesquisas proporcionam às empresas, bem como a otimização dos processos, evitando os desperdícios e gerando aumento nos lucros das mesmas.

A relação entre pesquisa e desenvolvimento empresarial é ratificada pela estatística de que os Estados Unidos e a China são líderes tanto em pesquisas bem como entre as maiores economias do mundo. Este fato se comprova através das pesquisas que relacionam os métodos de maior relevância para os países citados anteriormente. Tópicos como a Gestão de Processos e a Análise Multicritério auxiliam em um maior desenvolvimento industrial e econômico, pois possibilitam uma tomada de decisão mais precisa e com diminuição de riscos, além do aumento das chances de acerto.

Essa pesquisa possibilitou identificar as principais contribuições dos pesquisadores, o contexto em que os autores e seus respectivos trabalhos estão inseridos. A utilização dos 
softwares citados anteriormente otimiza o tempo do pesquisador, garantindo a confiabilidade e validação dos dados apresentados na pesquisa.

\section{REFERÊNCIAS}

ADUNLIN, G.et al. Multicriteria decision analysis in oncology. Health Expectations, v. 18, n. 6, p. 1812-1826, 2015.Acessoem: 19 mai. 2017.DOI: 10.1111/hex.12178

ARAÚJO, C. A. Bibliometria: evolução histórica e questões atuais. Em questão, Porto Alegre, v. $12, \quad$ n. $1, \quad$ p. 11-32, $2006 . \quad$ Disponível em:<http://seer.ufrgs.br/index.php/EmQuestao/article/view/16>. Acesso em: 23 mai. 2016.

BAGHERZADEH, G.et al. Developing a new approach for evaluation of business processes in a fuzzy environment. Technological and Economic Development of Economy, v. 22, n. 6, p. 783-807, 2016.Acessoem: 19 mai. 2017. DOI: 10.3846/20294913.2016.1221866

CONVERTINO, M. et al. Multi-criteria decision analysis to select metrics for design and monitoring of sustainable ecosystem restorations. Ecological indicators, v. 26, p. 76-86, 2013. Acessoem: 19 mai. 2017. DOI: 10.1016/j.ecolind.2012.10.005.

DA SILVEIRA GUEDES, V. L. A bibliometria e a gestão da informação e do Conhecimento científico e tecnológico: uma revisão da literatura. Ponto de Acesso, Salvador, v. 6, n. 2, p. 74-109, 2012. Disponível em: <https://portalseer.ufba.br/index.php/revistaici/article/view/5695>. Acesso em: 23 mai. 2016.

FERREIRA, A. G. C. Bibliometria na avaliação de periódicos científicos. Data Grama Zero, João Pessoa, v. 11, n. 3, p. 5, 2010. Disponível em: <http://basessibi.c3sl.ufpr.br/brapci/index.php/article/view/0000008927/738965b8bc4ee79ca9 007eef07685c93>. Acessoem: 24 mai. 2016.

GOMES, C. F. S. Principais características da teoria da utilidade multiatributo, e análise comparativa com a teoria da modelagem de preferências e teoria das expectativas. Artigo apresentado no Encontro Nacional de Engenharia de Produção, 1998. Disponível em: <http://abepro.org.br/biblioteca/ENEGEP1998_ART042.pdf>. Acesso em: 13 mai. 2016.

GUITOUNI, A.; MARTEL, J.M. Tentative guidelines to help choosing an appropriate MCDA method. European Journal of Operational Research,v. 109, n. 2, p. 501-521, 1998. Acessoem: 10 mai. 2016. DOI: 10.1016/S0377-2217(98)00073-3

KAPUR, P. K.; SINGH, J. N. P.; SINGH, O. Application of multi attribute utility theory in multiple releases of software. International Journal of System Assurance Engineering and Management, v. 6, n. 1, p. 61-70, 2015.Acesso em: 13 mai. 2016. DOI: 10.1007/s13198-0140243-4

KLANN, R. C. et al. Avaliação de desempenho das instituições de ensino superior pertencente á Associação Catarinense das Fundações Educacionais (ACAFE). Contabilidade, Gestão e Governança, Brasília, v. 15, n. 3, p.71-87, 2012. Disponível em:<https://cggamg.unb.br/index.php/contabil/article/view/363>. Acesso em: 20 jun. 2016. 
LUCAS, E. O.; GARCIA-ZORITA, J. C. Produção Científica sobre Capital Social: estudo por acoplamento bibliográfico. Em Questão, Porto Alegre, v. 20, n. 3, p. 27-42, 2014. Disponível em: <http://seer.ufrgs.br/index.php/EmQuestao/article/view/49122>. Acesso em: 16 ago. 2016.

MAIA, J. L.; DI SERIO, L. C.; FILHO, A. G. A. Pesquisa bibliométrica em estratégia como prática: resultados exploratórios e comparação de fontes. Revista Eletrônica Sistemas \& Gestão, Niterói, v. 10, n. 4, 2015. Disponível em: <http://www.revistasg.uff.br/index.php/sg/article/view/662/359>. Acesso em: 2 jun. 2016.

MINATOUR, Y.; BONAKDARI, H.; ALIAKBARKHANI, Z. S. Extension of Fuzzy Delphi AHP Based on Interval-Valued Fuzzy Sets and its Application in Water Resource Rating Problems. Water Resources Management, v. 30, n. 9, p. 3123-3141, 2016. Acesso em: 19 mai. 2017. DOI: $10.1007 / \mathrm{s} 11269-016-1335-5$

OGLE, R.A.; DEE, S.J.; COX, B. L. Resolving inherently safer design conflicts with decision analysis and multi-attribute utility theory. Process Safety and Environmental Protection, v. 97, p. 61-69, 2015.Acessoem: 19 mai. 2017. DOI: https://doi.org/10.1016/j.psep.2015.03.009

PORTER, M. E. Clusters and the new economics of competition. Boston: Harvard Business Review, 1998. Disponívelem:<http://clustermapping.us/sites/default/files/files/resource/Clusters_and_the_N ew_Economics_of_Competition.pdf $>$. Acesso em: 17 ago. 2016.

PRADABWONG, J.et al. Business process management and supply chain collaboration: a critical comparison. LogisticsResearch, v. 8, n. 1, p. 6, 2015. Acesso em: 18 mai. 2017. DOI: 10.1007/s12159-015-0123-6

LACERDA, R. T. de O. et al. Research Opportunities in Business Process Management and Performance Measurement from a Constructivist View. Knowledge and Process Management, v.23, n. 1, p. 18-30, 2015. Acesso em: 18 mai. 2017.DOI: 10.1002/kpm.1495

VAN DER AALST, W. M. P. Business process management: A comprehensive survey. ISRN Software Engineering, v. 2013, 2013. Acesso em: 13 mai. 2016. DOI: 10.1155/2013/507984

VAN ECK, N. J.; WALTMAN, L. Software survey: VOSviewer, a computer program for bibliometric mapping. Scientometrics, v. 84, n. 2, p. 523-538, 2010. Acesso em: 6 jun. 2016. DOI: $10.1007 / \mathrm{s} 11192-009-0146-3$

Como citar este documento: CHOAIRE, Gustavo Trindade et al. Análise da produção científica sobre Gestão de Processos. Revista Jovens Pesquisadores, Santa Cruz do Sul, v. 7 , n. 1, jan. 2017. ISSN 2237-048X. Disponível em: <https://online.unisc.br/seer/index.php/jovenspesquisadores/article/view/9275>. Acesso em: ... doi: http://dx.doi.org/10.17058/rjp.v7i1.9275. 